\title{
Impact of PercuSurge Device Conjugative With Intracoronary Administration of Nitroprusside on No-Reflow Phenomenon Following Primary Percutaneous Coronary Intervention
}

\author{
Ali A. Youssef, MD; Chiung-Jen Wu, MD*; Chi-Ling Hang, MD*; \\ Cheng-I Cheng, MD*; Cheng-Hsu Yang, MD*; Chien-Jen Chen, MD*; \\ Yen-Hsun Chen, MD*; Han-Tan Chai, MD*; Sarah Chua, MD*; \\ Kuo-Ho Yeh, MD*; Hon-Kan Yip, MD*
}

\begin{abstract}
Background The present study tested the hypothesis that when administered in conjunction with a PercuSurge device for treatment of acute myocardial infarction (AMI), intracoronary (IC) administration of nitroprusside (NTP) is safe and superior to IC administration of NTP alone or nitroglycerin (NTG) for reversing slow-flow or no-reflow, both of which occur frequently during primary percutaneous coronary intervention (PCI).

Methods and Results Sixty-two patients with ST-segment elevation AMI of $<12 \mathrm{~h}$ duration undergoing primary PCI were enrolled. When the final Thrombolysis In Myocardial Infarction (TIMI) flow was normal (TIMI-3), NTG 200 $\mathrm{g}$ was administered first, followed by (5 min later) NTP $100 \mu \mathrm{g}$ via an intra-guiding catheter. When

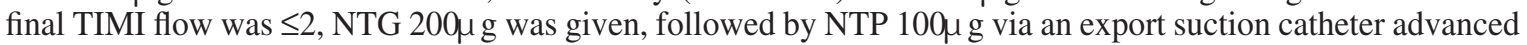
into the infract-related artery (IRA). Primary endpoint was epicardial blood flow (TIMI-flow), corrected TIMI frame counts, or microvascular circulation [myocardial blush (MB) grade]. Analytical results indicated that the final TIMI-3 flow was significantly higher in patients receiving NTP than in those receiving NTG therapy (100\% vs $88.7 \%$, $\mathrm{p}=0.023)$. As compared with NTG, NTP therapy significantly improved final MB grade $(\mathrm{p}<0.0001)$ and corrected TIMI flame count time $(\mathrm{p}<0.0001)$. Subgroup analysis demonstrated that final MB grade $(\mathrm{p}<0.001)$ and corrected TIMI flame count time $(\mathrm{p}<0.01)$ were significantly higher in patients $(\mathrm{n}=33)$ with than in patients $(n=29)$ without the PercuSurge. No significant NTP related adverse events occurred, apart from insignificant transient hypotension.

Conclusion IC administration of NTP is safe and superior to NTG for improving final epicardial blood flow and microvascular circulation in patients with AMI undergoing primary PCI. Combination therapy of PercuSurge device and NTP provided an additional benefit to NTP alone for improving microvascular circulation. (Circ J 2006; 70: $1538-1542$ )
\end{abstract}

Key Words: Nitroprusside; No-reflow; PercuSurge device; Slow-flow

$\mathbf{P}$ rimary percutaneous coronary intervention (PCI) significantly improves the survival of patients with acute myocardial infarction (AMI) and is superior to thrombolytic therapy in terms of immediate restoration of normal coronary flow in the infarct-related artery (IRA) and reduction of recurrent ischemia or reinfarction! 1,2 However, the benefit of primary PCI is limited by a $5-15 \%$ incidence of no-reflow! ${ }^{1-5}$ A body of evidence demonstrates that either slow-flow 4 [Thrombolysis In Myocardial Infarction (TIMI) grade 2 flow] in the IRA after reperfusion, which was previously regarded as successful angioplasty, 6 or noreflow ( $\leq$ TIMI grade 1 flow), ${ }^{4}$ is associated with relatively

(Received June 26, 2006; revised manuscript received September 12, 2006; accepted September 15, 2006)

Cardiology Department, Suez Canal University Hospital, Ismailia, Egypt, *Division of Cardiology, Chang Gung Memorial HospitalKaohsiung Medical Center, Chang Gung University Collage of Medicine, Kaohsiung, Taiwan, ROC

Mailing address: Hon-Kan Yip, MD, Division of Cardiology, Department of Internal Medicine, Chang Gung Memorial Hospital, Kaohsiung, 123, Ta Pei Road, Niao Sung Hsiang, Kaohsiung Hsien, 83301, Taiwan, ROC. E-mail: tang@ adm.cgmh.org.tw more extensive myocardial necrosis, and consequently left ventricular dilatation with poor regional and global contractile function and an untoward clinical outcome1,6,7,9-11 The mechanisms of slow-flow and no-reflow have been debated extensively, $412-16$ but a specific and efficacious method has not been suggested for promptly reversing slow-flow or noreflow in the IRA after primary PCI, $45-17$

The PercuSurge device has recently emerged as an attractive mechanical device that effectively restores normal coronary blood flow in the IRA, 5 , preventing both embolization in the microvasculature and no reflow, particularly in patients with high-burden thrombus formation (HBTF) in the IRA5,18 However, the benefit of this mechanical device is limited by a $5-10 \%$ incidence of no-reflow in patients with HBTF undergoing primary PCI5,18

An experimental study demonstrated that nitric oxide (NO) is a potent vasodilator in the resistance arteriolar circulation ${ }^{19}$ and plays a significant role in the control of coronary blood flow through the microcirculation. ${ }^{20}$ Nitroprusside (NTP), a direct donor of NO, has been demonstrated to be a derivation of NO with no requirement for prior intracellular metabolism? ${ }^{21}$ Additionally, a study has 
demonstrated that intracoronary (IC) administration of NTP is an effective, safe treatment for impaired blood flow and no-reflow during elective $\mathrm{PCI}^{22}$ However, the beneficial effects of this therapy on slow-flow or no-reflow in patients with AMI undergoing primary PCI is currently uncertain. Additionally, no data exists that addresses the impact of the PercuSurge device in conjunction with NTP therapy on additional benefits in reducing the slow-flow or no-reflow phenomenon following primary PCI.

Therefore, this study tested the hypothesis that when administered in conjunction with PercuSurge device for treatment of AMI, IC administration of NTP is safe and superior to IC administration of NTP alone or nitroglycerin (NTG) for reversing the slow-flow or no-reflow that frequently occurs during primary PCI.

\section{Methods}

\section{Patient Population and Inclusion Criteria}

At our hospital, all patients with ST-segment elevation AMI of $<12 \mathrm{~h}$ duration are eligible for primary PCI. The PercuSurge GuardWire ${ }^{\mathrm{TM}}$ device (Medtronic AVE, Santa Rosa, CA, USA) is used as a precaution against distal embolization in patients during primary PCI when angiographic results comply with the following criteria: (1) angiographic morphologic features of IRA showed HBTF as defined in our recent study; 4,5 (2) reference lumen diameter (RLD) of the IRA $\geq 3.5 \mathrm{~mm}$; and (3) AMI $<12 \mathrm{~h}$. Exclusion criteria were: (1) RLD of IRA $<3.5 \mathrm{~mm}$; (2) heavily calcified IRA; and (3) very tortuous IRA.

Because NTP is a strong vasodilatation agent, patients with systolic blood pressure $<90 \mathrm{mmHg}$ at the time of the final procedure before administration of NTP were excluded. Between November 2005 and April 2006, 62 patients who met the inclusion criteria were enrolled after giving informed consent. The Institutional Review Committee on Human Research at approved the study protocol.

\section{Procedure and Protocol}

A transradial approach using a $6 \mathrm{~F}$ arterial sheath has been the design protocol for AMI at our hospital since 2001.5 When diagnostic results demonstrated that the IRA met the criteria for a PercuSurge device therapy, the $6 \mathrm{~F}$ arterial sheath was replaced with a 7F arterial sheath and a 7F standard interventional guiding catheter was selectively positioned in the culprit artery. Thrombectomy with an export suction catheter was performed after inflation of the distal protection balloon prior to coronary angioplasty.

Clopidogrel $(300 \mathrm{mg}$ preoperative loading dose then $75 \mathrm{mg} /$ day) was given for at least 4 weeks to patients who underwent primary stenting. Aspirin (orally $100 \mathrm{mg} /$ day) was given to each patient indefinitely.

\section{Administration Route for NTG and NTP, Assessment of Epicardial Coronary Blood Flow and Microvascular Circulation}

When the final TIMI flow ${ }^{23}$ was normal (TIMI-3 flow), NTG 200 $\mathrm{g}$ was administered, followed immediately by angiographic examination. Repeat angiographic examination was performed $5 \mathrm{~min}$ later, followed by NTP $100 \mu \mathrm{g}$ administered via the intra-guiding catheter; angiographic examination was again immediately performed. A 5-min interval was used in this study to avoid any synergy between NTG and NTP on vasodilatation. In order to identify that the NTP effect was not transient, angiographic exami- nation was repeated 5 min after administration of NTP.

We proposed that a localized high concentration of either NTG or NTP would be more effective against the slowflow or no-reflow phenomenon. However, large dosages of these drugs are required to achieve to a localized therapeutic concentration if the drugs are administered via an intraguiding catheter over a short interval, and this may induce a severe hypotension, which is usually harmful to the patient. Thus, in order to avoid this unpredictable side-effect, we decided that when the final TIMI flow was $\leq 2$, a $6 \mathrm{~F}$ or $7 \mathrm{~F}$ export suction catheter would be used to advance into the IRA for administration of NTG and NTP. The patient's heart rate and blood pressure were continuously recorded during the procedure.

To compare the effect of NTG vs NTP on the epicardial coronary blood flow and integrity of the IRA microvasculature, the final TIMI flow grade, 23 corrected TIMI frame count (CTFC) ${ }^{24}$ and myocardial blush (MB) grade ${ }^{25}$ were assessed and consensus was reached among 3 interventional cardiologists immediately after primary PCI.

The primary endpoints were epicardial blood flow (TIMIflow), CTFC or MB grade, and the secondary endpoint was 30-day major adverse cardiac events (MACE) (defined as reinfarction, repeated target vessel revascularization, and 30-day death).

\section{Definitions and Data Collection}

AMI was defined as typical ischemic chest pain for $>30$ min with ST-segment elevation $>1 \mathrm{~mm}$ in 2 consecutive precordial or inferior leads. Unsuccessful reperfusion was defined as failure of the IRA to achieve normal blood flow ( $\leq$ TIMI-2 flow) or MB grade $\leq 1$. Slow-flow was defined as TIMI grade 2 flow and no-reflow was defined as $\leq$ TIMI grade 1 flow in the distal IRA in the absence of an occlusion at the treatment site or evidence of distal embolization occurring after successful coronary recanalization.

\section{Statistical Analysis}

Data are expressed as mean $\pm \mathrm{SD}$ or number $(\%)$ of patients. Continuous variables were compared using Student's t-test. Categorical variables were compared using the chisquare test for repeated measures. Statistical analysis was performed using SAS statistical software for Windows version 8.2 (SAS Institute, Cary, NC, USA). A value of $\mathrm{p}<0.05$ was considered statistically significant.

\section{Results}

Baseline Characteristics of the Study Patients (Table 1)

The mean patient age was 60 years. Of the 62 patients more than $90 \%$ were male. Low-density lipoprotein cholesterol levels, hypertension and current smoking were the most prevalent risk factors for coronary artery disease, followed by diabetes mellitus. Advanced Killip score $(\geq 3)$ was more than $17 \%$ upon presentation. More than half of the patients had an anterior wall infarction. A transradial artery approach was used for all patients. Angiographic examination showed more than $50 \%$ of the patients had multivessel disease. An intra-aortic balloon pump was used immediately before primary PCI in 6 patients who were in cardiogenic shock upon presentation because of an occlusion of the left anterior descending artery. Later in the procedure, these patients' blood pressure and condition were relatively stable. 
Table 1 Baseline Characteristics of 62 Study Patients With AMI

\begin{tabular}{lc}
\hline \hline & $(\%)$ or (mean \pm SD) \\
\hline Age (years) (mean \pm SD) (range: 31 to 84$)$ & $60.0 \pm 9.8$ \\
Male gender & $91.9 \%(57)$ \\
Diabetes mellitus & $22.6 \%(14)$ \\
Hypertension & $45.2 \%(28)$ \\
Hypercholesterolemia & $35.7 \%(22)$ \\
Low-density lipoprotein-cholesterol $>100 \mathrm{mg} /$ dl & $74.1 \%(46)$ \\
Current smoker & $41.9 \%(26)$ \\
Cardiogenic shock & $9.8 \%(6)$ \\
Killip score 3 & $8.1 \%(5)$ \\
Killip score $\leq 2$ & $82.3 \%(51)$ \\
Anterior wall myocardial infarction & $56.5 \%(35)$ \\
Transradial artery approach & $100 \%(62)$ \\
Multivessel disease & $53.2 \%(33)$ \\
Time from onset to first balloon dilatation (min) & $329 \pm 185$ \\
Infarct-related artery & \\
Left anterior descending artery & $56.5 \%(35)$ \\
Left circumflex artery & $11.3 \%(7)$ \\
Right coronary artery & $32.3 \%(20)$ \\
PercuSurge device utilization & $53.2 \%(33)$ \\
IIb/IIa receptor blockade use & $0 \%(0)$ \\
Stenting & $100 \%(62)$ \\
Intra-aortic balloon pump use & $9.8 \%(6)$ \\
Repeat target vessel revascularization & $0 \%(0)$ \\
Recurrent myocardial infarction & $0 \%(0)$ \\
30-day death & $3.2 \%(2)$ \\
\hline &
\end{tabular}

Data are mean $\pm S D$ or $\%$ (no.) of patients.

AMI, acute myocardial infarction.

Angiographic Results During Administration of NTG and NTP and Short-Term Outcomes (Tables 1-3)

Combined slow-flow and no-reflow occurred in $12.9 \%$ ( $\mathrm{n}=8$, including 4 patients with final TIMI flow $\leq 1$ ) following NTG therapy (Table 2). NTG 400 $\mathrm{g}$ was administered to these patients through the export suction catheter. All of these cases of slow-flow or no-reflow were immediately reversed by NTP administration via the export suction catheter. Only 3 of the 8 patients received more than $100 \mu \mathrm{g}$ of NTP (range 150-200 $\mathrm{g}$ ). Analysis showed that the final TIMI-3 flow and MB grade were significantly higher for IC NTP therapy than for IC NTG therapy. Moreover, compared with NTG treatment, NTP treatment resulted in a striking improvement of the CTFC. Thus, IC administration of NTP was more effective than IC administration of NTG for improving both epicardial blood flow and microvascular reperfusion. The final TIMI-3 flow, CTFC and MB grade did not differ immediately or at $5 \mathrm{~min}$ after IC administration of NTG or NTP (Table 2).

Table 3 shows the impact of the PercuSurge device on epicardial blood flow and microvascular circulation in patients undergoing primary PCI and IC administration of NTP. The final TIMI-3 flow did not differ between patients with and without use of the PercuSurge device for distal embolic protection. However, the MB grade was significantly increased and the CTFC significantly shortened in patients with the device than in those without it for distal embolic protection. Interestingly, the MB grade was markedly increased $(2.48 \pm 0.64$ vs $2.1 \pm 0.8, \mathrm{p}<0.001)$ and the CTFC was notably shortened $(15.4 \pm 8.7$ vs $19.5 \pm 15.2$, $\mathrm{p}<$ 0.001 ) for IC NTP therapy alone than for IC NTG therapy regardless of the use of the PercuSurge.

No repeat target vessel revascularization or reinfarction occurred during the 30-day clinical follow-up (Table 1). However, 2 patients who had cardiogenic shock upon pre-
Table 2 Final Angiographic Results of 62 Study Patients and Blood Pressure Following Intracoronary Administration of NTG and NPT

\begin{tabular}{lccc}
\hline \hline & Post NTG & Post NTP & $p$ value \\
\hline Final TIMI-3 flow & & & \\
$\quad$ Immediately & $87.1 \%(55)$ & $100 \%(62)$ & 0.023 \\
$\quad$ 5-min & $87.1 \%(55)$ & $100 \%(62)$ & 0.023 \\
Corrected TIMI frame count & & & \\
$\quad$ Immediately & $19.5 \pm 15.2$ & $13.0 \pm 7.6$ & $<0.0001$ \\
$\quad$ 5-min & $19.9 \pm 15.7$ & $13.6 \pm 7.9$ & $<0.0001$ \\
Myocardial blush grade & & & \\
$\quad$ Immediately & $2.1 \pm 0.8$ & $2.6 \pm 0.6$ & $<0.0001$ \\
$\quad$ 5-min & $2.1 \pm 0.8$ & $2.6 \pm 0.4$ & $<0.0001$ \\
Systolic blood pressure $(\mathrm{mmHg})$ & $112 \pm 14$ & $93 \pm 16$ & $<0.0001$
\end{tabular}

Data are mean $\pm S D$ or $\%$ (no.) of patients.

NTG, nitroglycerin; NTP, nitroprusside; TIMI, Thrombolysis In Myocardial Infarction.

Table 3 Impact of PercuSurge on Microvascular Circulation in Patients With IC Administration of NTP

\begin{tabular}{lccc}
\hline \hline & $\begin{array}{c}\text { PercuSurge } \\
(+) \\
(n=33)\end{array}$ & $\begin{array}{c}\text { PercuSurge } \\
(-) \\
(n=29)\end{array}$ & p value \\
\hline Final TIMI-3 flow & $100 \%(33)$ & $100 \%(29)$ & 1.0 \\
Corrected TIMI frame count & $11.2 \pm 6.2$ & $15.4 \pm 8.7$ & $<0.01$ \\
Myocardial blush grade & $2.75 \pm 0.42$ & $2.48 \pm 0.64$ & $<0.001$ \\
\hline
\end{tabular}

Data are mean $\pm S D$ or \% (no.) of patients.

IC, intracoronary. Other abbreviations see in Tables 1,2.

sentation died during this time, comorising 1 death from pumping failure and another from intracranial hemorrhage.

\section{Discussion}

This study, which examined the epicardial blood flow and microvascular circulation of the IRA in patients with AMI undergoing primary PCI has several striking clinical implications. First, NTP was more effective than NTG in improving final TIMI-3 flow, CTFC and MB grade of the IRA. Second, direct administration of NTP to the IRA via an export suction catheter, which was an original delivery method, was safe and superior to NTG for rapidly reversing slow-flow or no-reflow in patients undergoing primary PCI. Third, combined use of the PercuSurge device and IC administration of NTP was superior to NTP therapy alone for improving final microvascular circulation. Finally, the very low 30-day MACE in this study may reflect the efficacious result of NTP administration on the microvascular dysfunction that usually causes an unfavorable clinical outcome.

Although the mechanisms of slow-flow and no-reflow have been debated extensively in various clinical settings, ${ }^{4}, 12-16$ the significance of any single mechanism is not yet fully understood. Recent evidence suggests that slowflow or no-reflow is caused by microcirculation dysfunction at the level of the resistance arterioles 15,16 However, treatment of these phenomena remains problematic $4,15,16$ The principal finding of the present study is that IC administration of NTP, a direct donor of NO, was more effective than NTG in rapidly reversing slow-flow or non-reflow, both of which occur frequently during primary PCI. One study recently demonstrated that NTP is an effective and safe treatment for impaired blood flow and no-reflow associated with elective PCI ${ }^{22}$ Additionally, another recent study also demonstrated that NTP treatment resulted in a marked im- 
provement in both TIMI flow grade and CTFC in primary PCI patients 26 Thus, the results of our study are consistent with those obtained by those 2 studies ${ }^{22,26}$ suggesting that this is a novel therapeutic strategy for treating slow-flow or no-reflow following PCI in various clinical settings.

Importantly, NTP was superior to NTG in improving final TIMI-3 flow and MB grade $\geq 2$, and shortened the CTFC in patients undergoing primary PCI. Such distinctive findings can be reasonably explained as follow. First, it has been suggested that abnormal function of the resistance arterioles or pre-arterioles $(<200 \mu \mathrm{m}$ in diameter) plays an essential role in the no-reflow phenomenon!5,16,27 Metabolism of NTG by the vascular wall is required prior to derivation of NO; however, the resistance arterioles are unable to metabolize NTG to NO as do the large nonresistance epicardial vessels ${ }^{28}$ Additionally, although NTG is an effective vasodilator for large epicardial vessels, it is significantly less effective in the microvascular circulation.28 On the other hand, NO, which is derived from NTP without the requirement for prior intracellular metabolism ${ }^{21}$ is a potent vasodilator in the resistance arteriolar circulation 19,29 and plays a significant role in controlling blood flow through the coronary microcirculation. ${ }^{20,29}$ Furthermore, NO could positively affect latent collaterals or collateral blood flow by eliciting vasodilatation or inhibiting platelet aggregation in the vascular bed distal to the target lesion22,30 These mechanisms ${ }^{15,16,19-22,28-30}$ can explain why NTP was more effective than NTG in improving the final angiographic results. Second, recent studies have demonstrated that $\mathrm{HBTF}^{4}$ and lipid pool-like content ${ }^{31}$ in large IRAs are predictors of slow-flow or no-reflow, because distal macro- and microembolism of thrombi and plaque material frequently occurs in these vessels during balloon dilatation or stenting, which, in turn, induces severe microvascular dysfunction.,27,31 Thus, the pathophysiology of microvascular obstruction and dysfunction are proposed to be the result of microvascular plugging, microvascular spasm or edema, and platelet aggregation 32 Additionally, our recent study 5 demonstrated that the PercuSurge device is an effective tool for improving microvasculature integrity in large IRAs with HBTF. In the present study, a PercuSurge device was used to remove HBTF in more than $51 \%$ of the study patients. Moreover, microvasculature integrity was significantly improved with IC NTP therapy alone than with IC NTG therapy. Furthermore, the combination therapy of PercuSurge device and IC NTP was superior to IC NTP therapy only for improving microvasculature integrity during primary PCI. Accordingly, we suggest that the synergic effect of PercuSurge device, which plays an important role in preventing microvascular plugging, and IC NTP therapy, which relieves microvascular spasm, is crucial for preventing slow-flow or no-reflow phenomenon during primary PCI and is the principal reason for the improved final angiographic results. Finally, we used an export suction catheter to advance into the IRA for direct administration of NTP in cases of slow-flow or no-reflow in this study and we consider that this delivery method, which has not been previously reported, can provide a locally high concentration in IRA and thus induce extreme vasodilatation of the microvasculature. This novel administration approach may also explain why NTP produced a significantly higher final MB grade $(\geq 2)$ and a notably shorter CTFC than NTG in this clinical setting.

Experimental studies by Kloner et al $^{12}$ have demonstrated that the obstruction of capillaries is crucial for no-reflow phenomenon, and anatomical analysis indicates that capillaries do not have smooth muscle. Therefore, NTP can not reverse all of the factors that cause the no-reflow phenomenon. However, the findings from Kloner et al ${ }^{12}$ could, at least in part, explain why the MB of the IRA did not achieve grade 3 in all of the present patients.

The presence of no reflow is associated with a high risk of morbidity and mortality in patients undergoing primary PCI, during the procedure, and at short-term or long-term follow-up4,6,7,10,11 In the present study, the effect of IC NTP therapy for improving the final microvascular circulation was persistent. Additionally, the 30-day mortality rate was only $3.2 \%$. Such a low mortality rate is likely to be the result of successfully reversed slow-flow or no-reflow by the IC administration of NTP, which in turn, translated into a favorable clinical outcome. Furthermore, except for transient mild hypotension, IC administration of NTP did not elicit any other side-effect. Thus, these findings encourage the use of this therapeutic strategy for preventing and treating slow-flow or no-reflow during primary PCI in hemodynamically stable patients.

\section{Study Limitations}

First, adenosine and nicorandil have been shown to be effective for reversing slow-flow or no-reflow phenomenon during primary PCI33,34 However, without a comparison, we can not provide additional information that these drugs are superior to NTP or vice versa for reversing slow-flow or no-reflow phenomenon during primary PCI. Second, although the half life of NTG is very short ( $<2 \mathrm{~min})$ and the time period between NTG and NTP administration was $5 \mathrm{~min}$, this study can not completely rule out a synergic effect of the 2 drugs on vasodilatation. Finally, instead of using a conventional method for comparing a control group and study group, this study used a comparison of the final angiographic results for post-NTG therapy and post-NTP therapy in the same patient. However, this method provided the same variables and reliable baseline conditions for different pharmacological tests.

\section{Conclusion}

IC administration of NTP is safe and appears to have a much more powerful effect than IC administration of NTG for reversing slow-flow or no-reflow and improving final epicardial flow and microvascular circulation in patients with AMI undergoing primary PCI.

\section{References}

1. Grines CL, Browne KF, Marco J, Rothbaum D, Stone GW, O'Keefe I, et al. A comparison of primary angioplasty with thrombolytic therapy for acute myocardial infarction. N Engl J Med 1993; 328: 673-679.

2. Stone GW, Grines CL, Browne KF, Marco J, Rothbaum D, O'Keefe $\mathrm{J}$, et al. Predictors of in-hospital and 6 month outcome after acute myocardial infarction in the reperfusion era: The Primary Angioplasty in Myocardial Infarction (PAMI) trial. J Am Coll Cardiol 1995; 25: $370-377$.

3. Ito H, Okamura A, Iwakura K. Myocardial perfusion patterns related to thrombolysis in myocardial infarction perfusion grades after coronary angioplasty in patients with acute anterior wall myocardial infarction. Circulation 1996; 93: $1993-1999$.

4. Yip HK, Chen MC, Chang HW, Hang CL, Hsieh YK, Fang CY, et al. Angiographic morphologic features of infarct-related arteries and timely reperfusion in acute myocardial infarction: Predictors of slowflow and no-reflow. Chest 2002; 122: $1322-1332$.

5. Yip HK, Wu CJ, Chang HW, Fang CY, Yang CH, Chen SM, et al. Effect of the PercuSurge GuardWire Device on the integrity of 
microvasculature and clinical outcomes during primary transradial coronary intervention in acute myocardial infarction. Am J Cardiol 2003; 92: $1331-1335$.

6. Stone GW, Grines CL, Browne KF, Marco J, Rothbaum D, O'Keefe $\mathrm{J}$, et al. Predictors of in-hospital and 6 month outcome after acute myocardial infarction in the reperfusion era: The Primary Angioplasty in Myocardial Infarction (PAMI) trial. J Am Coll Cardiol 1995; 25: $370-377$.

7. Yip HK, Wu CJ, Chang HW, Chen MC, Hang CL, Fang CY, et al. Comparison of impact of primary percutaneous transluminal coronary angioplasty and primary stenting on short-term mortality in patients with cardiogenic shock and evaluation of prognostic determinants. Am J Cardiol 2001; 87: 1184-1188.

8. Sklenar J, Ismail S, Villanueva FS, Goodman C, Glasheen WP, Kaul S. Dobutamine echocardiography for determining the extent of myocardial salvage after reperfusion: An experimental evaluation. Circulation 1994; 90: $1502-1512$.

9. Kern MJ, Moore JA, Aguirre FV, Bach RG, Caracciolo EA, Wolford $\mathrm{T}$, et al. Determination of angiographic (TIMI grade) blood flow by intracoronary doppler flow velocity during acute myocardial infarction. Circulation 1996; 94: 1545-1552.

10. Ito H, Maruyama A, Iwakura K, Takiuchi S, Masuyama T, Hori M, et al. Clinical implications of "no reflow" phenomenon: A predictor of complications and left ventricular remodeling in reperfused anterior wall myocardial infarction. Circulation 1996; 93: 223-228.

11. Morishima I, Sone T, Okumura K, Tsuboi H, Kondo J, Mukawa H, et al. Angiographic no-reflow phenomenon as a predictor of adverse long-term outcome in patients with percutaneous transluminal coronary angioplasty for first acute myocardial infarction. $J$ Am Coll Cardiol 2003; 36: $1202-1209$.

12. Kloner RA, Ganote CE, Jennings RB. The "no-reflow" phenomenon following temporary coronary occlusion in the dog. J Clin Invest 1974; 54: 1496-1508.

13. Baim DS, Carrozza JP. Understanding the "No-Reflow" problem. Cathet Cardiovasc Diagn 1996; 39: 7-8.

14. Abbo KM, Dooris M, Glazier S, O'Neill WW, Byrd D, Grines CL, et al. Features and outcome of no-reflow after percutaneous coronary intervention. Am J Cardiol 1995; 75: 778-782.

15. Kern MJ, Eeckhout E. The coronary no-reflow phenomenon: A review of mechanism and therapies. Eur Heart J 2001; 22: 729-739.

16. Reffelmann T, Kloner RA. The "no-reflow" phenomenon: Basic science and clinical correlates. Heart 2002; 87: 162-168.

17. Maekawa K, Kawamoto K, Fuke S, Yoshioka R, Saito H, Sato T, et al. Effects of intraaortic balloon pumping on the angiographic noreflow phenomenon after percutaneous coronary intervention in patients with anterior myocardial infarction. Circ J 2006; 70: 37-43.

18. Umeda H, Katoh $\mathrm{T}$, Iwase $\mathrm{M}$, Izawa $\mathrm{H}$, Nagata $\mathrm{K}$, Watanabe $\mathrm{K}$, et al. The distal protection during primary percutaneous coronary intervention alleviates the adverse effect of large thrombus burden on myocardial reperfusion. Circ J 2006; 70: 232-238.

19. Myers PR, Bannitt P, Guerra R, Harrison D. Characteristics of canine coronary resistance arterioles: Importance of the endothelium. Am J
Physiol 1989; 257: H603-H610.

20. Kuo L, Chilian W, Davis M. Interaction of pressure-induced and flow-induced response in porcine coronary resistance vessels. Am J Physiol 1991; 261: H1706-H1715.

21. Bates J, Baker M, Guerra R, Harrison D. Nitric oxide generation from nitroprusside by vascular tissue: Evidence that reduction of the nitroprusside anion and cyanide loss are required. Biochem Pharmacol 1991; 42: S157-S165.

22. Hillegass WB, Dean NA, Liao L, Rhinehart RG, Myers PR. Treatment of no-reflow and impaired flow with the nitric oxide donor nitroprusside following percutaneous coronary interventions: Initial human clinical experience. J Am Coll Cardiol 2001; 37: 1335-1343.

23. The TIMI Study Group. The Thrombolysis in Myocardial Infarction (TIMI) Trial: Phase 1 findings. N Engl J Med 1985: 312: 932-936.

24. Gibson CM, Cannon CP, Daley WL, Cannon CP, Daley WL, Dodge JT, et al. TIMI frame count: A quantitative method for assessing coronary artery flow. Circulation 1996; 93: 879-888.

25. Van't Hof AWJ, Liem A, Suryapranata H, Hoorntje JCA, de Boer MJ, Zijlstra F. Angiographic assessment of myocardial reperfusion in patients treated with primary angioplasty for acute myocardial infarction: Myocardial blush grade. Circulation 1998; 97: 2302-2306.

26. Pasceri V, Pristipino C, Pelliccia F, Granatelli A, Speciale G, Roncella A, et al. Effects of the nitric oxide donor nitroprusside on no-reflow phenomenon during coronary interventions for acute myocardial infarction. Am J Cardiol 2005; 95: 1358-1361.

27. Rezkalla SH, Kloner RA. No-reflow phemononen. Circulation 2002; 105: 656-662.

28. Kurz M, Lamping K, Bates J, Easthma C, Marcus M, Harrison D. Mechanisms responsible for heterogeneous coronary microvascular response to nitroglycerin. Circ Res 1991; 68: 847-855.

29. Parham WA, Bouhasin A, Ciaramita JP, Khoukaz S, Hermann SC, Kern MJ. Coronary hyperemic dose response of intracoronary sodium nitroprusside. Circulation 2004; 109: 1236-1243.

30. Traverse J, Kinn J, Klassen C, Duncker D, Bache R. Nitric oxide inhibition impairs blood flow during exercise in heart with a collateraldependent myocardial region. J Am Coll Cardiol 1997; 31: 67-74.

31. Tanaka A, Kawarabayashi T, Nishibori Y, Sano T, Nishida Y, Fukuda $\mathrm{D}$, et al. No-reflow phenomenon and lesion morphology in patients with acute myocardial infarction. Circulation 2002; 105: $2148-$ 2152 .

32. Hori M, Inoue M, Kitakaze M, Koretsune Y, Iwai K,Tamai J, et al. Role of adenosine in hyperemic response of coronary blood flow in microembolization. Am J Physiol 1986; 250: H509-H518.

33. Ozdemir M, Cemri M, Yalcin R, Cengel A. Use of intracoronary adenosine for the management of slow-no-reflow phenomenon during percutaneous interventions. Cathet Cardiovasc Interv 2001; 54: 267-268.

34. Lim SY, Bae EH, Jeong MH, Kang DG, Lee YS, Kim KH, et al. Effect of combined intracoronary adenosine and nicorandil on no-reflow phenomenon during percutaneous coronary intervention. Circ J 2004; 68: $928-932$. 